\title{
Boundary Integral Equations in Frequency Domain for Interface Linear Cracks under Impact Loading
}

\author{
Oleksandr V. Menshykov", Marina V. Menshykova, Igor A. Guz \\ Centre for Micro- and Nanomechanics (CEMINACS) \\ School of Engineering, University of Aberdeen \\ Aberdeen AB24 3UE, Scotland, UK
}

\begin{abstract}
The linear crack between two dissimilar elastic isotropic half-spaces under normal pulse loading is considered. The system of boundary integral equations for displacements and tractions in the frequency domain is derived from the dynamic Somigliana identity and adapted to solve the problem in the time domain. The numerical convergence of the method with respect to the number of the Fourier coefficients is proved. The effects of material properties of the bimaterial on the distribution of stress intensity factors (opening and transverse shear modes) are presented and analysed.
\end{abstract}

Keywords: interface crack, bimaterial, impact loading, boundary integral equations, stress intensity factor.

"Corresponding author. Tel.: +44 1224 273326; fax: +43 1224 272519;

e-mail: o.menshykov@abdn.ac.uk 


\section{Introduction and state-of-the-art of the problem}

The problems devoted to dynamic loading of engineering materials with cracklike defects are traditionally of interest in academia and industry, as these defects could be the main reason of structures' collapse. Solving any crack problems, the crack faces contact interaction must be taken into account, as it changes the distribution of the stresses and the displacements in the vicinity of the crack not only quantitatively but also qualitatively. However there are difficulties in solving the contact problems because singular and hypersingular integrals appear and should be regularised and computed.

In paper [1] two approaches to solve the contact problem for harmonic longitudinal loading were compared. For the plane problem solution the iterative algorithm and boundary element method in time and frequency domains were used. Numerical results were obtained for crack opening, contact forces and dynamic stress intensity factors. Contact crack problems for oblique incidence of harmonic waves were considered in [2, 3]. The results for the linear crack [2] and the penny-shaped crack [3] were obtained for various values of the angle of wave incidence, the wave frequency and the friction coefficient. Efficient and accurate technique for solving the three-dimensional contact problems for cracked solids was presented in [4], where the problems of normal incidence of tension-compression and shear waves on a penny-shaped crack were solved. Elliptical crack without initial opening was considered in [5], where the contact interaction of the crack faces was taken into account for the crack under normally incident tension-compression wave. 
Complex loading of a rectilinear crack in homogeneous material under three different contact conditions was studied in [6] by Ostrik, who considered smooth, sliding slip and adhesion between the crack faces. Interfacial crack under shear loading with dynamic anti-plane traction was also considered by Matbuly [7], who used the variables' separation technique in order to derive the singular system of integral equations.

At the same time, special attention must be paid to the response of cracked materials to non-harmonic loading, particularly impact loading. The impact load generates the elastic waves in the structure causing the local stresses to increase beyond their corresponding values under static loads of the same magnitude [8]. The transient dynamic two-dimensional crack analysis for anisotropic materials is given in [9]. The paper presents the comparison of two hypersingular time-domain boundary element methods.

Finite elastic cracked members under impact loading were also considered in $[10,11]$. In the study the computation of the critical intersection angle for straight and curved cracks as well as investigation of the influence of free surface on the distribution of stress intensity factor along the crack-front were presented. The solution was obtained by implementing 3D time-domain formulation of boundary element method.

The approach presented in [11] could be extended to study free surface effects for running crack in finite elastic solids. Itou [12] presented the research on 3D impact response of the cylindrical crack under longitudinal stress waves. For the solution of the incident shear stress wave problem the method employed in [12] could be used. 
Rectangular and penny shaped cracks under impact loading were considered in [13-15]. For the cracks in infinite elastic solid the dependence of the dynamic stress intensity factors on the type and direction of incident waves was investigated.

Furthermore, with the increasing use of composite materials in engineering applications understanding the fracture mechanism in different types of composites becomes more and more important. A number of researchers paid special attention to the crack problems in layered composites and bimaterials. The elastic wave scattering by a doubly periodic array of planar delaminations of arbitrary shape presented in [16]. The authors used the approach, which is the extension of the boundary integral equation method, to solve the problem. As an example of the method, solutions for two types of cracks, rectangular and elliptic, were obtained.

Penny-shaped interface crack under unit pressure was considered in [17], where the study employs crack opening and sliding as primary unknowns, that allows the determination of crack opening and sliding displacement as well as complex stress intensity factors. Harmonic loading of penny-shaped crack in multi-layered composite was studied by Yu and Cooper [18]. They used a point force method to obtain results for the crack under harmonic loading.

Additionally torsion harmonic loading of penny-shaped crack in layered composite was studied by Mykhaskiv and Stankevych [19] using boundary integral equation method. In the paper the effects of wave number, material properties, and the crack interfaces distance on the dynamic stress intensity factor were investigated. The problem of penny-shaped crack and its interaction 
with a thin interlayer joining two elastic half-spaces when subjected to a timeharmonic tensile loading was solved by the improved boundary integral equation method in [20].

Contact problems for interlayer cracks were considered in [21, 22]. The problems of linear crack [21] and penny-shaped crack [22] between two dissimilar elastic isotropic half-spaces under harmonic loading were solved. The multi-parametric algorithm was presented and the system of boundary integral equations for displacements and tractions was derived to study the effect of frequency of the loading on the stress intensity factors.

The problems of impact loading of cracked materials were considered by number of authors. Elastic cracked material under transient loading was considered in [23]. Boundary integral equations in the frequency domain were used to solve the problem, presenting the components of the solution by the Fourier exponential series. Two-dimensional problem of interface crack in layered anisotropic solids was solved in [24]. In the paper the time-domain boundary element method in conjunction with a multi-domain technique was developed for to solve the problem. The effects of the crack configuration, the material anisotropy, the layer combination and the dynamic loading on the dynamic stress intensity factors and the scattered elastic wave fields were investigated.

The interfacial cracks in orthotropic materials were studied in [25]. In the paper the stress intensity factor history around the crack-tips was obtained for the normal and shear impact loading. The study of interface crack under antiplane shear impact load was made by Li and Tai in [26]. 
Bimaterials with interface crack under impact loading were studied in [27] numerically and experimentally. The analysis of the energy release rate, stress intensity factors, crack-opening-displacements dependence on the time and the phase angle was given.

The problem for a penny-shaped interface crack was solved in [28], where the torsional impact response was investigated. Torsional impact load of a penny-shaped crack situated between two elastic media was also considered in [29]. In the studies the problem was reduced to the solution of the pair of dual integral equations using Laplace and Hankel transforms. The expression for the stress intensity factor at the tip of the crack was obtained.

The current study is devoted to the application of the method and techniques developed for the contact crack problems in the frequency domain $[1-5,21,22,30]$ for the case of transient loading. The method was initially adopted for homogeneous materials under normal impact loading [23] and further extended here for interface cracks in bimaterials. The major advantage of the method proposed is that there is no need for direct and inverse Laplace transforms to solve the problem. The method also may be used to account for the crack faces contact interaction and its effects on stresses in the vicinity of the crack.

\section{Problem formulation}

Bi-material with finite length crack at the interface is considered. The bi-material consists of two homogeneous isotropic half-spaces $\Omega^{(1)}$ and $\Omega^{(2)}$ with dissimilar properties. 
The stress-strain state is defined by Lamé equations for both domains (in the absence of body forces)

$$
\begin{gathered}
\left(\lambda^{(m)}+\mu^{(m)}\right) \operatorname{grad} \operatorname{div} \mathbf{u}^{(m)}(\mathbf{x}, t)+\mu^{(m)} \Delta \mathbf{u}^{(m)}(\mathbf{x}, t)=\rho^{(m)} \partial_{t}^{2} \mathbf{u}^{(m)}(\mathbf{x}, t), \mathbf{x} \in \Omega^{(m)}, \\
t \in \mathrm{T}=[0, \infty),
\end{gathered}
$$

where $\mathbf{u}^{(m)}(\mathbf{x}, t)$ is the displacement vector, $\Delta$ is the Laplace operator, $\lambda^{(m)}$ and $\mu^{(m)}$ are the Lamé elastic constants, $\rho^{(m)}$ is the specific material density.

At the bonding interface the following conditions of continuity for displacements and stresses must be satisfied:

$$
\mathbf{u}^{(1)}(\mathbf{x}, t)=\mathbf{u}^{(2)}(\mathbf{x}, t), \quad \mathbf{p}^{(1)}(\mathbf{x}, t)=-\mathbf{p}^{(2)}(\mathbf{x}, t), \quad \mathbf{x} \in \Gamma^{*}, \quad t \in \mathrm{T},
$$

where

$$
\Gamma^{*}=\Gamma^{(1)} \cap \Gamma^{(2)}, \Gamma^{\mathrm{cr}}=\Gamma^{(1) \mathrm{cr}} \cup \Gamma^{(2) \mathrm{cr}},
$$

and the traction vectors on the crack surface are

$$
\mathbf{p}^{(1)}(\mathbf{x}, t)=\mathbf{g}^{(1)}(\mathbf{x}, t), \quad \mathbf{p}^{(2)}(\mathbf{x}, t)=\mathbf{g}^{(2)}(\mathbf{x}, t), \quad \mathbf{x} \in \Gamma^{(m) c r}, \quad t \in \mathrm{T} .
$$

In the case of elastic solid occupying the whole space, the Sommerfeld radiation-type condition, which provides a finite elastic energy of an infinite body, is also imposed at infinity on the vector of displacements:

$$
\|\mathbf{u}(\mathbf{x}, t)\| \leq c / r,
$$

where $c$ is a constant and $r \rightarrow \infty$ is the distance from the origin.

We assume that the initial displacements of the points of the body are absent. The components of displacement field in both half-spaces can be represented in terms of boundary displacements and tractions by using the Somigliana dynamic identity [30]: 


$$
\begin{gathered}
u_{j}^{(m)}(\mathbf{x}, t)=\int_{T} \int_{\Gamma^{(m)}}\left(p_{i}^{(m)}(\mathbf{y}, \tau) U_{i j}^{(m)}(\mathbf{x}, \mathbf{y}, t-\tau)-u_{i}^{(m)}(\mathbf{y}, \tau) W_{i j}^{(m)}(\mathbf{x}, \mathbf{y}, t-\tau)\right) d \mathbf{y} d \tau, \\
\mathbf{x} \in \Omega^{(m)}, \quad t \in T, \quad j=1,2,
\end{gathered}
$$

where $\Gamma^{(1)}$ and $\Gamma^{(2)}$ are regular surfaces, $\mathbf{x}$ is the point of observation, $y$ is the point of loading and $U_{i j}^{(m)}(\mathbf{x}, \mathbf{y}, t-\tau)$ is the Green's fundamental displacement tensor:

$$
U_{i j}^{(m)}(\mathbf{x}, \mathbf{y}, t-\tau)=\frac{1}{2 \pi \mu^{(m)}}\left(\psi^{(m)} \delta_{i j}-\chi^{(m)} \frac{\left(y_{i}-x_{i}\right)}{r} \frac{\left(y_{j}-x_{j}\right)}{r}\right) .
$$

Here $\delta_{i j}$ is the Kronecker delta, $r$ is the distance between the observation point and the load point, and the functions $\psi^{(m)}$ and $\chi^{(m)}$ in the time-domain can be represented as the combinations of the Heaviside function and the Dirac delta functions, which for the 2D case considered have the following form:

$$
\begin{gathered}
\psi^{(m)}=K_{0}\left(l_{2}^{(m)}\right)+\frac{1}{l_{2}^{(m)}}\left[K_{1}\left(l_{2}^{(m)}\right)-\frac{c_{2}^{(m)}}{c_{1}^{(m)}} K_{1}\left(l_{1}^{(m)}\right)\right] \\
\chi^{(m)}=K_{2}\left(l_{2}^{(m)}\right)-\left(\frac{c_{2}^{(m)}}{c_{1}^{(m)}}\right)^{2} K_{2}\left(l_{1}^{(m)}\right),
\end{gathered}
$$

with the modified Bessel function $K_{n}(\bullet)$ (the second kind and order $n$ ) [31]; and the longitudinal and the transversal waves velocities $l_{1}^{(m)}=i \omega r / c_{1}^{(m)}$, $l_{2}^{(m)}=i \omega r / c_{2}^{(m)}, c_{1}^{(m)}=\sqrt{\left(\lambda^{(m)}+2 \mu^{(m)}\right) / \rho^{(m)}}$ and $c_{2}^{(m)}=\sqrt{\mu^{(m)} / \rho^{(m)}}$.

To obtain the integral kernel $W_{i j}^{(m)}(\mathbf{x}, \mathbf{y}, t-\tau)$ we must apply differential operator

$$
P_{i k}[\bullet,(\mathbf{y})]=\lambda n_{i}(\mathbf{y}) \frac{\partial[\bullet]}{\partial y_{k}}+\mu\left[\delta_{i k} \frac{\partial[\bullet]}{\partial \mathbf{n}(\mathbf{y})}+n_{k}(\mathbf{y}) \frac{\partial[\bullet]}{\partial y_{i}}\right]
$$


to $U_{i j}^{(m)}(\mathbf{x}, \mathbf{y}, t-\tau)$. Doing so we will get

$$
\begin{aligned}
& W_{i j}^{(m)}(\mathbf{x}, \mathbf{y}, t-\tau)=\lambda^{(m)} n_{i}^{(m)}(\mathbf{y}) \frac{\partial}{\partial y_{k}} U_{k j}^{(m)}(\mathbf{x}, \mathbf{y}, t-\tau)+ \\
& \mu^{(m)} n_{k}^{(m)}(\mathbf{y})\left[\frac{\partial}{\partial y_{k}} U_{i j}^{(m)}(\mathbf{x}, \mathbf{y}, t-\tau)+\frac{\partial}{\partial y_{i}} U_{k j}^{(m)}(\mathbf{x}, \mathbf{y}, t-\tau)\right] .
\end{aligned}
$$

When we apply differential operator (10) to the Somigliana identity, then for both half-spaces the components of the traction vector can be obtained in terms of boundary displacements and traction:

$$
\begin{gathered}
p_{j}^{(m)}(\mathbf{x}, t)=\int_{T} \int_{\Gamma^{(m)}}\left(p_{i}^{(m)}(\mathbf{y}, \tau) K_{i j}^{(m)}(\mathbf{x}, \mathbf{y}, t-\tau)-u_{i}^{(m)}(\mathbf{y}, \tau) F_{i j}^{(m)}(\mathbf{x}, \mathbf{y}, t-\tau)\right) d \mathbf{y} d \tau, \\
\mathbf{x} \in \Omega^{(m)}, \quad t \in T, \quad j=1,2
\end{gathered}
$$

The operator (10) with respect to $\mathbf{x}$ is used to obtain integral kernels $K_{i j}^{(m)}(\mathbf{x}, \mathbf{y}, t-\tau)$ and $F_{i j}^{(m)}(\mathbf{x}, \mathbf{y}, t-\tau)$ from $U_{i j}^{(m)}(\mathbf{x}, \mathbf{y}, t-\tau)$ and $W_{i j}^{(m)}(\mathbf{x}, \mathbf{y}, t-\tau)$.

With the assumption of smoothness of the boundary displacements and traction vectors, the boundary integral equations for the limiting case $\mathbf{x} \rightarrow \Gamma^{(m)}$ can have the form:

$$
\begin{aligned}
& \frac{1}{2} u_{j}^{(m)}(\mathbf{x}, t)=\int_{T} \int_{\Gamma^{(m)}}\left(p_{i}^{(m)}(\mathbf{y}, \tau) U_{i j}^{(m)}(\mathbf{x}, \mathbf{y}, t-\tau)-u_{i}^{(m)}(\mathbf{y}, \tau) W_{i j}^{(m)}(\mathbf{x}, \mathbf{y}, t-\tau)\right) d \mathbf{y} d \tau, \\
& \frac{1}{2} p_{j}^{(m)}(\mathbf{x}, t)=\int_{T} \int_{\Gamma^{(m)}}\left(p_{i}^{(m)}(\mathbf{y}, \tau) K_{i j}^{(m)}(\mathbf{x}, \mathbf{y}, t-\tau)-u_{i}^{(m)}(\mathbf{y}, \tau) F_{i j}^{(m)}(\mathbf{x}, \mathbf{y}, t-\tau)\right) d \mathbf{y} d \tau,
\end{aligned}
$$

where $\mathbf{x} \in \Gamma^{(m)}, \quad t \in T$.

On the surfaces $\Gamma^{(m), c r}$ and $\Gamma^{(m)^{*}}$ the boundary integral equations have the following form: 


$$
\begin{aligned}
& \frac{1}{2} g_{j}^{(m)}(\mathbf{x}, t)=\int_{T} \int_{\Gamma^{(m) c r}}\left(g_{i}^{(m)}(\mathbf{y}, \tau) K_{i j}^{(m)}(\mathbf{x}, \mathbf{y}, t-\tau)-u_{i}^{(m)}(\mathbf{y}, \tau) F_{i j}^{(m)}(\mathbf{x}, \mathbf{y}, t-\tau)\right) d \mathbf{y} d \tau+ \\
& \int_{T} \int_{\Gamma^{(m) c r}}\left(p_{i}^{(m)}(\mathbf{y}, \tau) K_{i j}^{(m)}(\mathbf{x}, \mathbf{y}, t-\tau)-u_{i}^{(m)}(\mathbf{y}, \tau) F_{i j}^{(m)}(\mathbf{x}, \mathbf{y}, t-\tau)\right) d \mathbf{y} d \tau, \quad \mathbf{x} \in \Gamma^{(m) c r}, \\
& \frac{1}{2} p_{j}^{(m)}(\mathbf{x}, t)=\int_{T} \int_{\Gamma^{(m) c r}}\left(g_{i}^{(m)}(\mathbf{y}, \tau) K_{i j}^{(m)}(\mathbf{x}, \mathbf{y}, t-\tau)-u_{i}^{(m)}(\mathbf{y}, \tau) F_{i j}^{(m)}(\mathbf{x}, \mathbf{y}, t-\tau)\right) d \mathbf{y} d \tau+ \\
& \int_{T} \int_{\Gamma^{(m) c r}}\left(p_{i}^{(m)}(\mathbf{y}, \tau) K_{i j}^{(m)}(\mathbf{x}, \mathbf{y}, t-\tau)-u_{i}^{(m)}(\mathbf{y}, \tau) F_{i j}^{(m)}(\mathbf{x}, \mathbf{y}, t-\tau)\right) d \mathbf{y} d \tau, \quad \mathbf{x} \in \Gamma^{(m)^{*}}, \\
& \frac{1}{2} u_{j}^{(m)}(\mathbf{x}, t)=\int_{T} \int_{\Gamma^{(m)}}\left(g_{i}^{(m)}(\mathbf{y}, \tau) U_{i j}^{(m)}(\mathbf{x}, \mathbf{y}, t-\tau)-u_{i}^{(m)}(\mathbf{y}, \tau) W_{i j}^{(m)}(\mathbf{x}, \mathbf{y}, t-\tau)\right) d \mathbf{y} d \tau+ \\
& \int_{T} \int_{\Gamma^{(m)}}\left(p_{i}^{(m)}(\mathbf{y}, \tau) U_{i j}^{(m)}(\mathbf{x}, \mathbf{y}, t-\tau)-u_{i}^{(m)}(\mathbf{y}, \tau) W_{i j}^{(m)}(\mathbf{x}, \mathbf{y}, t-\tau)\right) d \mathbf{y} d \tau, \quad \mathbf{x} \in \Gamma^{(m) c r}, \\
& \frac{1}{2} u_{j}^{(m)}(\mathbf{x}, t)=\int_{T} \int_{\Gamma^{(m)}}\left(g_{i}^{(m)}(\mathbf{y}, \tau) U_{i j}^{(m)}(\mathbf{x}, \mathbf{y}, t-\tau)-u_{i}^{(m)}(\mathbf{y}, \tau) W_{i j}^{(m)}(\mathbf{x}, \mathbf{y}, t-\tau)\right) d \mathbf{y} d \tau+ \\
& \int_{T} \int_{\Gamma^{(m)}}\left(p_{i}^{(m)}(\mathbf{y}, \tau) U_{i j}^{(m)}(\mathbf{x}, \mathbf{y}, t-\tau)-u_{i}^{(m)}(\mathbf{y}, \tau) W_{i j}^{(m)}(\mathbf{x}, \mathbf{y}, t-\tau)\right) d \mathbf{y} d \tau, \quad \mathbf{x} \in \Gamma^{(m)^{*}},
\end{aligned}
$$

where $m=1,2$.

To simplify the system (15)-(18) we can introduce the following new variables

$$
\begin{aligned}
& u_{i}^{*}(\mathbf{x}, t)=u_{i}^{(1)}(\mathbf{x}, t), \quad p_{i}^{*}(\mathbf{x}, t)=-p_{i}^{(1)}(\mathbf{x}, t), \quad \mathbf{x} \in \Gamma^{(1)^{*}}, t \in T, \\
& u_{i}^{*}(\mathbf{x}, t)=u_{i}^{(2)}(\mathbf{x}, t), \quad p_{i}^{*}(\mathbf{x}, t)=p_{i}^{(2)}(\mathbf{x}, t), \quad \mathbf{x} \in \Gamma^{(2)^{*}}, t \in T,
\end{aligned}
$$

and take into account that on the interface we integrate over the surface $\Gamma^{*}$ with the outer normal vector $\mathbf{n}_{\Gamma^{*}}=\mathbf{n}_{\Gamma^{(2) *}}=-\mathbf{n}_{\Gamma^{(1)^{*}}}$ As the result we can get a system of boundary integral equations which is simpler than the system (15)-(18), as it does not contain integral kernels $K_{i j}^{(m)}(\mathbf{x}, \mathbf{y}, t-\tau)$ and $F_{i j}^{(m)}(\mathbf{x}, \mathbf{y}, t-\tau)$. Consequently, there are no hypersingular integrals to be regularised and calculated: 


$$
\begin{aligned}
& -\int_{T} \int_{\Gamma^{(1) \mathrm{cr}}} g_{i}^{(1)}(\mathbf{y}, \tau) U_{i j}^{(1)}(\mathbf{x}, \mathbf{y}, t-\tau) d \mathbf{y} d \tau=-\frac{1}{2} u_{i}^{(1)}(\mathbf{x}, t)-\int_{T} \int_{\Gamma^{(1) \mathrm{cr}}} u_{i}^{(1)}(\mathbf{y}, \tau) W_{i j}^{(1)}(\mathbf{x}, \mathbf{y}, t-\tau) d \mathbf{y} d \tau+ \\
& \int_{T} \int_{\Gamma^{*}} u_{i}^{*}(\mathbf{y}, \tau) W_{i j}^{(1)}(\mathbf{x}, \mathbf{y}, t-\tau) d \mathbf{y} d \tau-\int_{T} \int_{\Gamma^{*}} p_{i}^{*}(\mathbf{y}, \tau) U_{i j}^{(1)}(\mathbf{x}, \mathbf{y}, t-\tau) d \mathbf{y} d \tau, \quad \mathbf{x} \in \Gamma^{(1) \mathrm{cr}}, \\
& -\int_{T} \int_{\Gamma^{(2) c r}} g_{i}^{(2)}(\mathbf{y}, \tau) U_{i j}^{(2)}(\mathbf{x}, \mathbf{y}, t-\tau) d \mathbf{y} d \tau=-\frac{1}{2} u_{i}^{(2)}(\mathbf{x}, t)-\int_{T} \int_{\Gamma^{(2) c r}} u_{i}^{(2)}(\mathbf{y}, \tau) W_{i j}^{(2)}(\mathbf{x}, \mathbf{y}, t-\tau) d \mathbf{y} d \tau+ \\
& \int_{T} \int_{\Gamma^{*}} u_{i}^{*}(\mathbf{y}, \tau) W_{i j}^{(2)}(\mathbf{x}, \mathbf{y}, t-\tau) d \mathbf{y} d \tau+\int_{T} \int_{\Gamma^{*}} p_{i}^{*}(\mathbf{y}, \tau) U_{i j}^{(2)}(\mathbf{x}, \mathbf{y}, t-\tau) d \mathbf{y} d \tau, \quad \mathbf{x} \in \Gamma^{(2) \mathrm{cr}}, \\
& -\int_{T} \int_{\Gamma^{(1) c r}} g_{i}^{(1)}(\mathbf{y}, \tau) U_{i j}^{(1)}(\mathbf{x}, \mathbf{y}, t-\tau) d \mathbf{y} d \tau=-\frac{1}{2} u_{i}^{*}(\mathbf{x}, t)-\int_{T} \int_{\Gamma^{(1) c r}} u_{i}^{(1)}(\mathbf{y}, \tau) W_{i j}^{(1)}(\mathbf{x}, \mathbf{y}, t-\tau) d \mathbf{y} d \tau+ \\
& \int_{T} \int_{\Gamma^{*}} u_{i}^{*}(\mathbf{y}, \tau) W_{i j}^{(1)}(\mathbf{x}, \mathbf{y}, t-\tau) d \mathbf{y} d \tau-\int_{T} \int_{\Gamma^{*}} p_{i}^{*}(\mathbf{y}, \tau) U_{i j}^{(1)}(\mathbf{x}, \mathbf{y}, t-\tau) d \mathbf{y} d \tau, \quad \mathbf{x} \in \Gamma^{*}, \\
& -\int_{T} \int_{\Gamma^{(2) c r}} g_{i}^{(2)}(\mathbf{y}, \tau) U_{i j}^{(2)}(\mathbf{x}, \mathbf{y}, t-\tau) d \mathbf{y} d \tau=-\frac{1}{2} u_{i}^{*}(\mathbf{x}, t)-\int_{T} \int_{\Gamma^{(2) c r}} u_{i}^{(2)}(\mathbf{y}, \tau) W_{i j}^{(2)}(\mathbf{x}, \mathbf{y}, t-\tau) d \mathbf{y} d \tau+ \\
& \int_{T} \int_{\Gamma^{*}} u_{i}^{*}(\mathbf{y}, \tau) W_{i j}^{(2)}(\mathbf{x}, \mathbf{y}, t-\tau) d \mathbf{y} d \tau+\int_{T} \int_{\Gamma^{*}} p_{i}^{*}(\mathbf{y}, \tau) U_{i j}^{(2)}(\mathbf{x}, \mathbf{y}, t-\tau) d \mathbf{y} d \tau, \quad \mathbf{x} \in \Gamma^{*} .
\end{aligned}
$$

It shall be also added that the method can be applied to solve $2 \mathrm{D}$ and 3D problems for cracks of arbitrary geometry (e.g., cracks and delaminations between layers of composite pipes). Then the major issue is the derivation of fundamental solutions $U_{i j}^{(m)}(\mathbf{x}, \mathbf{y}, t-\tau)$ and $W_{i j}^{(m)}(\mathbf{x}, \mathbf{y}, t-\tau)$, or $K_{i j}^{(m)}(\mathbf{x}, \mathbf{y}, t-\tau)$ and $F_{i j}^{(m)}(\mathbf{x}, \mathbf{y}, t-\tau)$, because the differential operator (10) contains components of the normal vector to the crack's surface and combinations of partial directional derivatives with respect to different directions. For the case of plane (3D) or linear/straight (2D) crack the derivations are greatly simplified. Thus, in the current study authors decided to focus on a single linear interface crack only.

Please note that even for the case of impact loading the components of the stress-strain state could be expanded into the Fourier series [23, 30, 32, 33]: 


$$
f(\bullet, t)=\operatorname{Re}\left\{\sum_{k=-\infty}^{+\infty} f^{k}(\bullet) e^{i \omega_{k} t}\right\},
$$

where $\omega_{k}=2 \pi k / T$, and the appropriate Fourier coefficients are given as

$$
f^{k}(\bullet)=\frac{\omega}{2 \pi} \int_{0}^{T} f(\bullet, t) e^{-i \omega_{k} t} d t
$$

Taking aforementioned into account we can rewrite the system of equations (21)-(24) into the following one:

$$
\begin{gathered}
-\int_{\Gamma^{(1) \mathrm{cr}}} g_{i}^{k,(1)}(\mathbf{y}) U_{i j}^{(1)}\left(\mathbf{x}, \mathbf{y}, \omega_{k}\right) d \mathbf{y}=-\frac{1}{2} u_{i}^{k,(1)}(\mathbf{x})-\int_{\Gamma^{(1) \mathrm{cr}}} u_{i}^{k,(1)}(\mathbf{y}) W_{i j}^{(1)}\left(\mathbf{x}, \mathbf{y}, \omega_{k}\right) d \mathbf{y}+ \\
\int_{\Gamma^{*}} u_{i}^{k, *}(\mathbf{y}) W_{i j}^{(1)}\left(\mathbf{x}, \mathbf{y}, \omega_{k}\right) d \mathbf{y}-\int_{\Gamma^{*}} p_{i}^{k, *}(\mathbf{y}) U_{i j}^{(1)}\left(\mathbf{x}, \mathbf{y}, \omega_{k}\right) d \mathbf{y}, \quad \mathbf{x} \in \Gamma^{(1) \mathrm{cr}}, \\
-\int_{\Gamma^{(2) c \mathrm{cr}}} g_{i}^{k,(2)}(\mathbf{y}) U_{i j}^{(2)}\left(\mathbf{x}, \mathbf{y}, \omega_{k}\right) d \mathbf{y}=-\frac{1}{2} u_{i}^{k,(2)}(\mathbf{x})-\int_{\Gamma^{(2) c r}} u_{i}^{k,(2)}(\mathbf{y}) W_{i j}^{(2)}\left(\mathbf{x}, \mathbf{y}, \omega_{k}\right) d \mathbf{y}- \\
\int_{\Gamma^{*}} u_{i}^{k, *}(\mathbf{y}) W_{i j}^{(2)}\left(\mathbf{x}, \mathbf{y}, \omega_{k}\right) d \mathbf{y}+\int_{\Gamma^{*}} p_{i}^{k, *}(\mathbf{y}) U_{i j}^{(2)}\left(\mathbf{x}, \mathbf{y}, \omega_{k}\right) d \mathbf{y}, \quad \mathbf{x} \in \Gamma^{(2) \mathrm{cr}}, \\
-\int_{\Gamma^{(1) c r}} g_{i}^{k,(1)}(\mathbf{y}) U_{i j}^{(1)}\left(\mathbf{x}, \mathbf{y}, \omega_{k}\right) d \mathbf{y}=-\frac{1}{2} u_{i}^{k, *}(\mathbf{x})-\int_{\Gamma^{(1) c r}} u_{i}^{k,(1)}(\mathbf{y}) W_{i j}^{(1)}\left(\mathbf{x}, \mathbf{y}, \omega_{k}\right) d \mathbf{y}+ \\
\int_{\Gamma^{*}} u_{i}^{k, *}(\mathbf{y}) W_{i j}^{(1)}\left(\mathbf{x}, \mathbf{y}, \omega_{k}\right) d \mathbf{y}-\int_{\Gamma^{*}} p_{i}^{k, *}(\mathbf{y}) U_{i j}^{(1)}\left(\mathbf{x}, \mathbf{y}, \omega_{k}\right) d \mathbf{y}, \quad \mathbf{x} \in \Gamma^{*}, \\
-\int_{\Gamma^{(2) c r}} g_{i}^{k,(2)}(\mathbf{y}) U_{i j}^{(2)}\left(\mathbf{x}, \mathbf{y}, \omega_{k}\right) d \mathbf{y}=-\frac{1}{2} u_{i}^{k, *}(\mathbf{x})-\int_{\Gamma^{(2) c r}} u_{i}^{k,(2)}(\mathbf{y}) W_{i j}^{(2)}\left(\mathbf{x}, \mathbf{y}, \omega_{k}\right) d \mathbf{y}- \\
\int_{\Gamma^{*}} u_{i}^{k, *}(\mathbf{y}) W_{i j}^{(2)}\left(\mathbf{x}, \mathbf{y}, \omega_{k}\right) d \mathbf{y}+\int_{\Gamma^{*}} p_{i}^{k, * *}(\mathbf{y}) U_{i j}^{(2)}\left(\mathbf{x}, \mathbf{y}, \omega_{k}\right) d \mathbf{y}, \quad \mathbf{x} \in \Gamma^{*},
\end{gathered}
$$

with the Fourier complex-valued coefficients of the known tractions at the crack surface $g_{i}^{k,(m)}(\mathbf{x})$, unknown tractions $p_{i}^{k, *}(\mathbf{x})$ and displacements at the interface and the crack surface $u_{i}^{k, *}(\mathbf{x})$ and $u_{i}^{k,(m)}(\mathbf{x})$. 
Using a piecewise-constant approximation of the known and unknown functions we can obtain from the system of boundary integral equations (27)(30) the system of linear algebraic equations (please, see paper [34] for details).

It may be added that the detailed investigation of the solution's convergence with respect to the number and size of the boundary elements and the number of time intervals has been presented in [2] and [21] for linear crack in homogeneous and layered materials, so here we followed the recommendations suggested in [21]. Although the bonding interface extends to infinity, the outer discretization can be truncated at certain distance (in this paper - 4 half-lengths of the crack) to the crack tip, because the elements that are far from the crack have very little effect on the solution near the tip (please, see [34]).

Note that the non-integrable singularities in the integral kernels of boundary integral equations (27)-(30) which rank exceeds the dimension of the integration region makes the appropriate integrals singular. Consequently, they must be treated in the sense of the Hadamard finite parts (please see [2, 30]).

The special attention must also be paid to the number of Fourier coefficients used to approximate the solution and initial. Thus, according to [23] for the impact (or sharp pulse) loading the number of Fourier coefficients shall be at least 30 coefficients to adequately approximate the Heaviside pulse. Some details of the analysis of the convergence of the solution with respect to the number of Fourier coefficients are presented in the current study.

Thus to use the methodology and results which the authors obtained previously in the frequency domain [21, 34-36], the external transient dynamic 
load has been approximated by the Fourier exponential series. Hence, the Heaviside impact pulse was approximated by the trapezoidal stress pulse

$$
\sigma(t)=\sigma^{*}\left\{\begin{array}{l}
\frac{t}{t^{*}}\left(H(t)-H\left(t-t^{*}\right)\right)+\left(H\left(t-t^{*}\right)-H\left(t-t^{*}-t_{d}\right)\right) \\
+\left(2-\frac{t-t_{d}}{t^{*}}\right)\left(H\left(t-t^{*}-t_{d}\right)-H\left(t-2 t^{*}-t_{d}\right)\right)
\end{array}\right\},
$$

where $c_{2} t^{*}=0.1$ and $c_{2} t_{d}=12$ [23], and the latter one was approximated by the Fourier series for different numbers of the Fourier coefficients.

\section{Numerical results}

We will consider the following numerical example: $2 a$ length linear crack under the unit intensity impact pulse of normal incidence. The half-spaces materials' properties:

\begin{tabular}{|l|c|c|c|}
\hline Material & $\begin{array}{c}\text { Young's } \\
\text { modulus }\end{array}$ & Poisson ratio & Density \\
\hline Steel & $200 \mathrm{GPa}$ & 0.25 & $7800 \mathrm{~kg} / \mathrm{m}^{3}$ \\
\hline Aluminium & $70 \mathrm{GPa}$ & 0.35 & $2700 \mathrm{~kg} / \mathrm{m}^{3}$ \\
\hline Epoxy & $4.6 \mathrm{GPa}$ & 0.36 & $1380 \mathrm{~kg} / \mathrm{m}^{3}$ \\
\hline Portland concrete & $40 \mathrm{GPa}$ & 0.20 & $2230 \mathrm{~kg} / \mathrm{m}^{3}$ \\
\hline Lead & $14 \mathrm{GPa}$ & 0.42 & $1134 \mathrm{~kg} / \mathrm{m}^{3}$ \\
\hline
\end{tabular}

To validate the method the crack on steel-steel interface was considered and the results were compared with the solution obtained for the homogeneous problem in [23] and the model solution obtained for the Heaviside stress pulse 
by Gross and Zhang [13]. The dynamic stress intensity factor (opening mode only, as the shear mode is absent for the normal pulse loading in homogeneous material) normalized by the corresponding static value (obtained for the static tension of the unit magnitude) is given in Figure 1, where the model numerical solution is also presented.

The dynamic stress intensity factors were computed in the vicinity of the crack tip using the following expressions $[37,38]$ :

$$
\begin{aligned}
& K_{I}^{\max }=\max _{t} \lim _{r \rightarrow 0} p_{n}^{*}(R+r, t) \sqrt{2 \pi r}, \\
& K_{I I}^{\max }=\max _{t} \lim _{r \rightarrow 0} p_{\tau}^{*}(R+r, t) \sqrt{2 \pi r} .
\end{aligned}
$$

Where $p_{n}^{*}(R+r, t), p_{\tau}^{*}(R+r, t)$ are normal and tangential components of the traction vector at the bonding interface, $r$ is the distance from the crack tip.

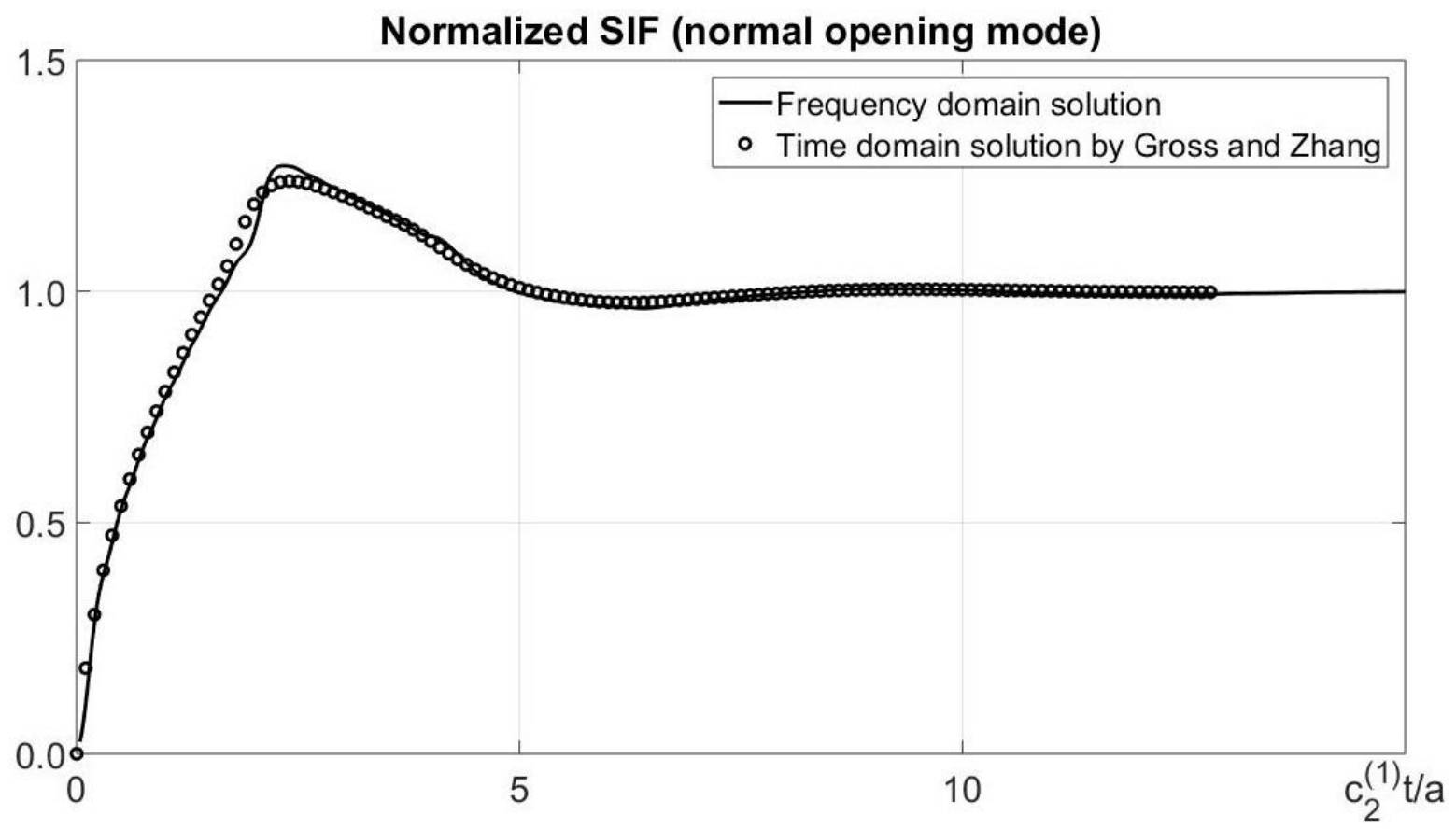

Fig. 1. Normalized stress intensity factor (normal opening mode) in time for homogeneous material 
As one can see the results are in a very good agreement. Consequently, the proposed method can be used for the case of transient impact loading (as was expected based on the results presented in [23]).

The convergence of the method for different numbers of the Fourier coefficients used to approximate the loading pulse and the stresses and displacements of the crack faces was analyzed, and the distribution of the normalized dynamic stress intensity factors (normal opening and transverse shear modes) in time is given in Figures 2 and 3 for Steel-Aluminium interface. One can clearly see the numerical convergence of the method (for both modes) with the increase in the number of coefficients used. Thus, in the following computations for different combinations of the materials of the half-spaces we decided to use 50 Fourier coefficients (solid lines in Figures 2 and 3).

Finally, normalized dynamic stress intensity factors (normal opening and transverse shear modes) in time for different bimaterials (Steel-Aluminium, Steel-Epoxy, Steel-Concrete and Steel-Lead) are given in Figures 4 and 5.

It shall be noted that the response time and the magnitude of the stress intensity factors clearly depend on the materials' properties significantly shifting in time for the materials with lower values of the Young's modulus (as Lead and Epoxy in the examples provided). 


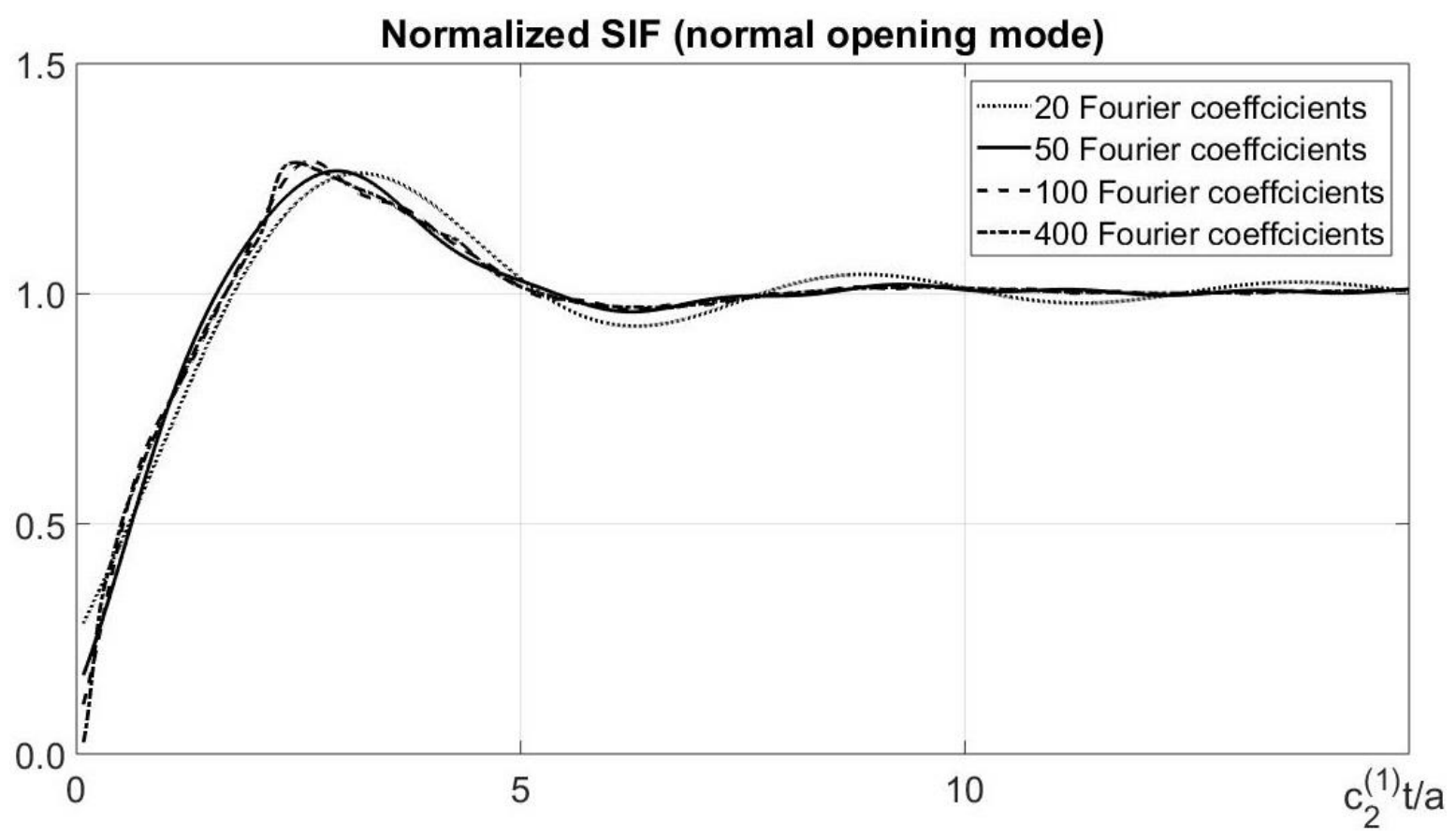

Fig. 2. Normalized stress intensity factors (normal opening mode) in time for different Fourier coefficients' numbers

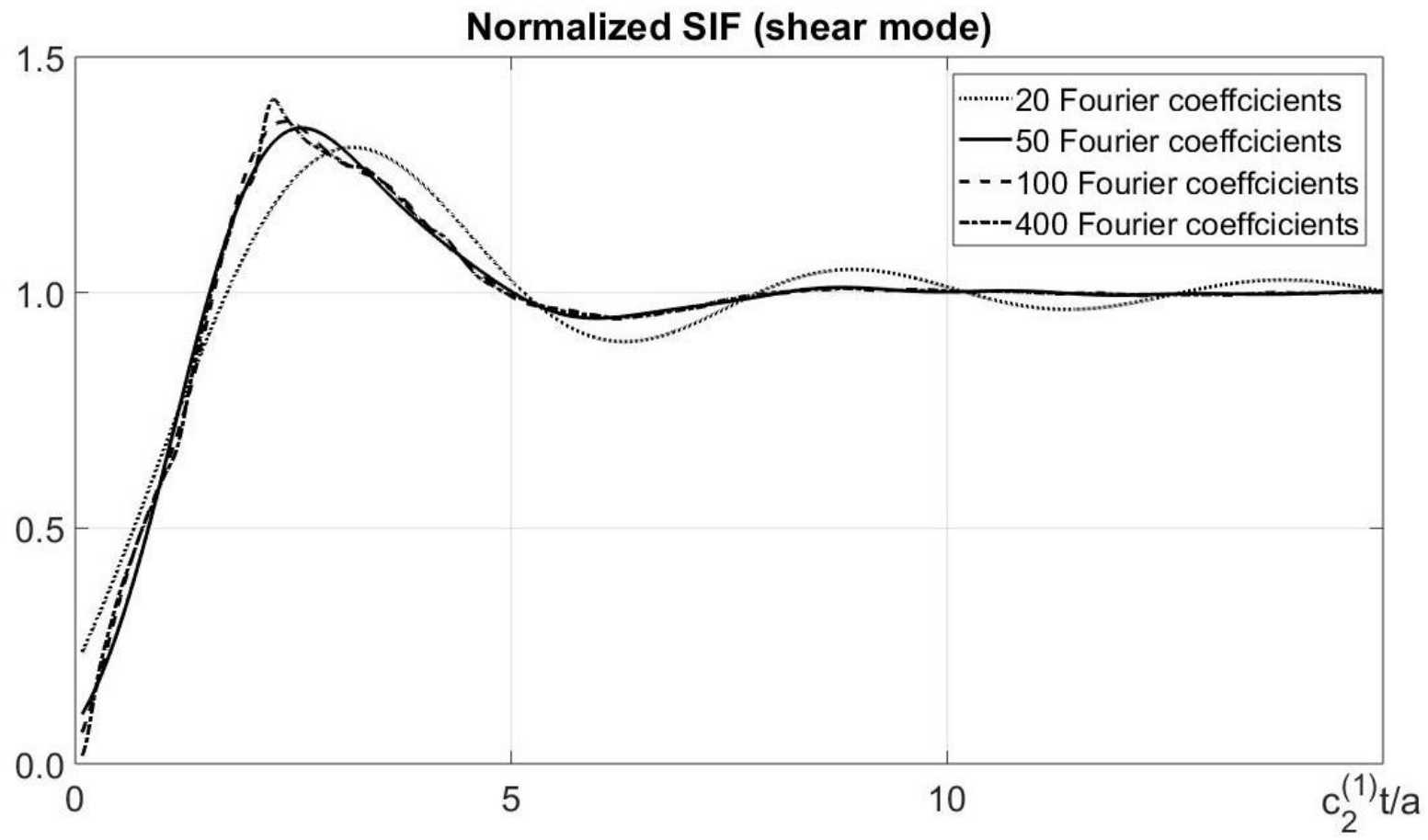

Fig. 3. Normalized stress intensity factors (shear mode) in time for different Fourier coefficients' numbers 


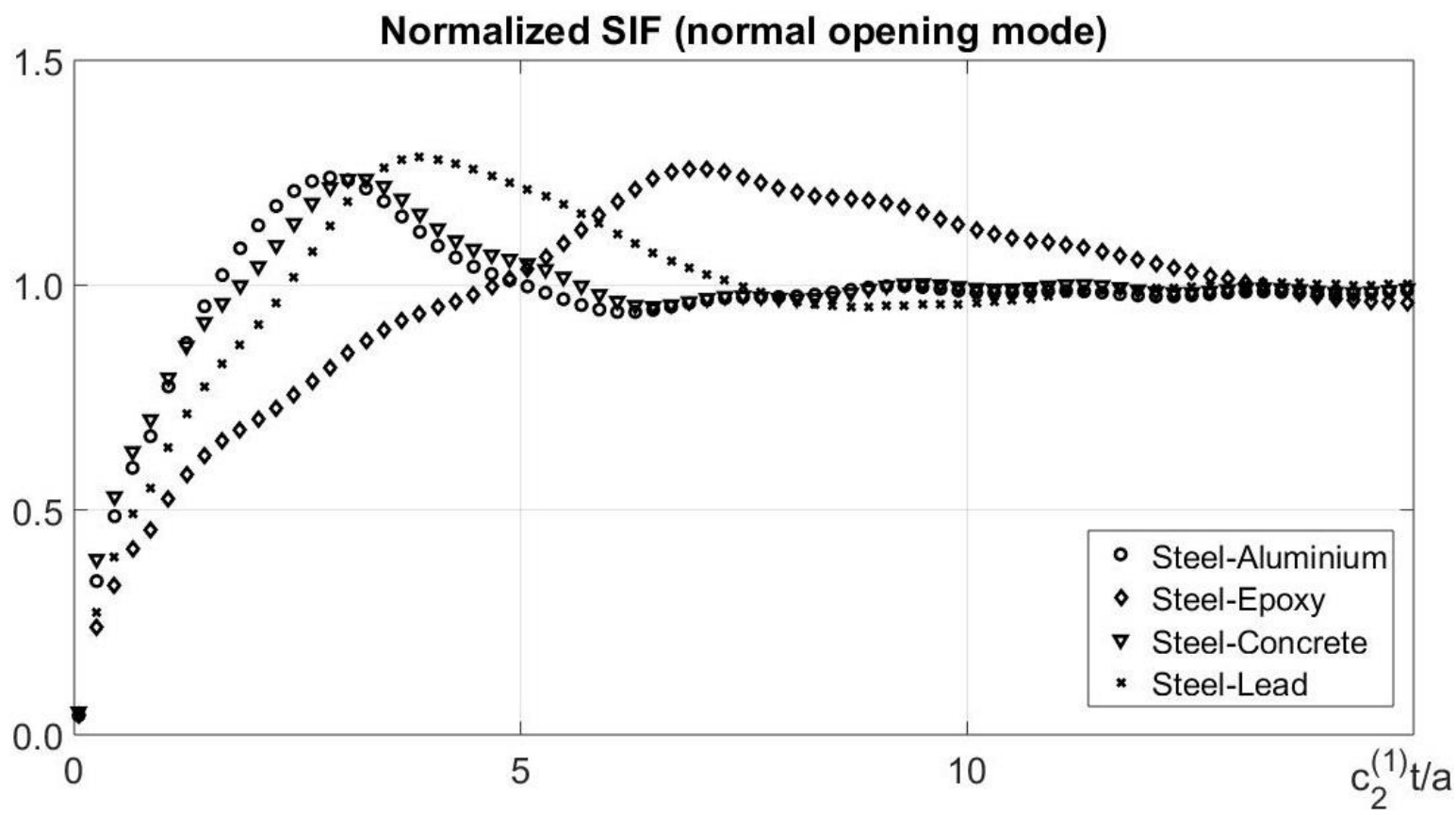

Fig. 4. Normalized stress intensity factors (normal opening mode) in time for different materials combinations

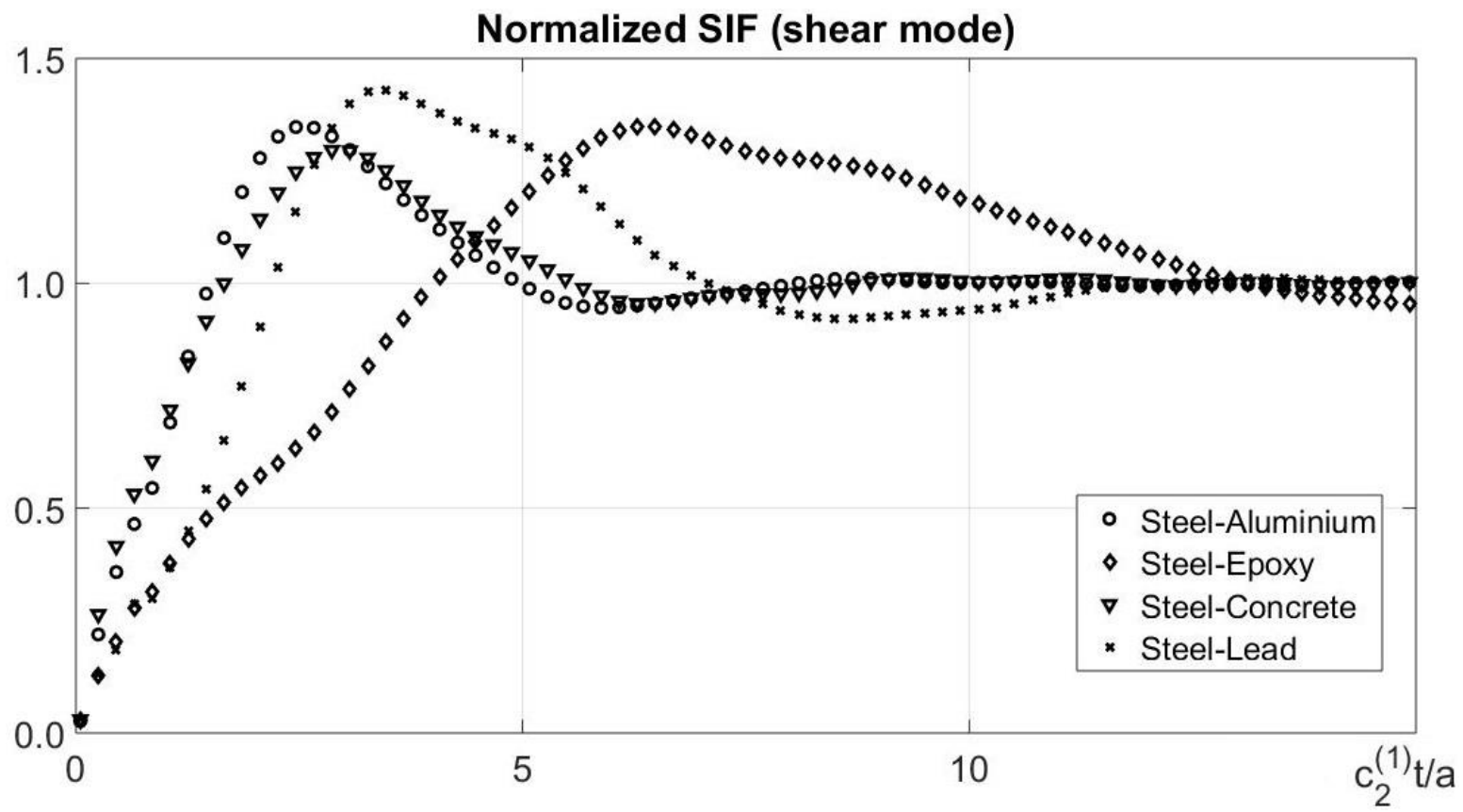

Fig. 5. Normalized stress intensity factor (shear mode) in time for different materials combinations 


\section{Conclusions}

In the paper the boundary integral equations method in the frequency domain was successfully used to solve the problem for an interface linear crack under normal Heaviside pulse loading. The convergence of results was analysed and the results were validated through the comparison with the results for homogeneous case.

It shall be also noted that for oblique pulse loading or any loading including the shear component (e.g. normal shear pulse) the opposite crack faces inevitably would be in contact and, as it was mentioned in the first section of this paper, the crack closure and friction will significantly change the solution of the problem. Therefore, the taking these effects into account shall be the next stage of the research (either for cracked homogenous or composite materials). The first results for the linear crack in homogeneous material under normal impact loading have been recently presented in [39] with particular attention to the effects of friction between contacting faces of the crack.

\section{References}

[1] A. Czekanski, V. Zozulya, Comparative study of time and frequency domain approaches in contact problem for the I-mode crack under harmonic loading, Engineering Analysis with Boundary Elements, 2018, 95, 200-214.

[2] O. Menshykov, M. Menshykova, I.A. Guz, Effect of friction of the crack faces for a linear crack under an oblique harmonic loading, International Journal of Engineering Science, 2008, 46, 438-458. 
[3] O. Menshykov, I. Guz, Contact interaction of crack faces under oblique incidence of a harmonic wave, International Journal of Fracture, 2006, 139, $145-152$.

[4] O. Menshykov, I.A. Guz, Effect of contact interaction of the crack faces for a crack under harmonic loading, International Applied Mechanics, 2007, 43(7), 809-815.

[5] A.N. Guz, O.V. Menshykov, V.V. Zozulya, Surface contact of elliptical crack under normally incident tension-compression wave, Theoretical and Applied Fracture Mechanics, 2003, 40(3), 285-291.

[6] V.I. Ostrik, Contact of faces of a rectilinear crack under complex loading and various contact conditions, Acta Mechanica, 2019, 230, 3741-3758.

[7] M.S. Matbuly, Analytical solution for an interfacial crack subjected to dynamic anti-plane shear loading, Acta Mechanica, 2006, 184, 77-85.

[8] E.P. Chen, G.C. Sih, Transient response of cracks to impact loads, Mechanics of Fracture 4: Elastodynamic Crack Problems (Edited by GC Sih), Noordhoff, Leyden, 1977, 1-58.

[9] M. Wuensche, Ch. Zhang, F. Garcia-Sanchez, A. Saez, J. Sladek, V. Sladek, On two hypersingular time-domain BEM for dynamic crack analysis in 2D anisotropic elastic solids, Computational Methods in Applied Mechanics and Engineering, 2009, 198, 2812-2824.

[10] A.K. Agrawal, A study of free surface effects on moving cracks under impact loading by BEM, Engineering Analysis with Boundary Elements, 2002, 26, 253-264. 
[11] A.K. Agrawal, N.N. Kishore, A study of free surface effects on through cracks under impact loading, Engineering Analysis with Boundary Elements, $2001,25,391-405$.

[12] S. Itou, Dynamic stress intensity factors around a cylindrical crack in an infinite elastic medium subject to impact load, International Journal of Solids and Structures, 2007, 44, 7340-7356.

[13] Ch. Zhang, D. Gross, On wave propagation in elastic solids with cracks (Advances in Fracture Mechanics), WIT Press, 1997.

[14] Ch. Zhang, D. Gross, Transient elastodynamic analysis of a penny-shaped crack, Engineering Fracture Mechanics, 1993, 46, 641-654.

[15] B. Chen, D. Gross, Dynamic stress intensity factors of a penny-shaped crack subjected to an annular impact, Journal of Applied Mathematics and Mechanics, 1998, 78(12) , 861-864.

[16] M. Golub, O. Doroshenko, Boundary integral equation method for simulation scattering of elastic waves obliquely incident to a doubly periodic array of interface delaminations, Journal of Computational Physics, 2019, $376,675-693$.

[17] B. Kilic, E. Madenci, Hypersingular integral equations for the solution of penny-shaped interface crack problems, Journal of Mechanics of Materials and Structures, 2007, 2(4), 729-751.

[18] H.Y. Yu, K.P. Cooper, Dynamic penny-shaped cracks in multilayer sandwich composites, Theoretical and Applied Fracture Mechanics, 2009, $51,181-188$. 
[19] V. Mykhaskiv, V. Stankevych, Elastodynamic problem for a layered composite with penny-shaped crack under harmonic torsion, Z Angew Math Mech (ZAMM), 2019, DOI: 10.1002/zamm.201800193.

[20] V. Mykhaskiv, V. Stankevych, I. Zhbadynskyi, Ch. Zhang, 3-D dynamic interaction between a penny-shaped crack and a thin interlayer joining two elastic half-spaces, International Journal of Fracture, 2009, 159, 137-149.

[21] M. Menshykova, O. Menshykov, I.A. Guz, An iterative BEM for the dynamic analysis of interface crack contact problems, Engineering Analysis with Boundary Elements, 2011, 35, 735-749.

[22] V. Menshykov, O. Menshykov, I.A. Guz, Interfacial crack between elastic half-spaces under harmonic loading, International Applied Mechanics, 2007, 43(8), 865-873.

[23] M. Menshykova, O. Menshykov, I.A. Guz, M. Wuensche, Ch. Zhang, A boundary integral equations method in the frequency domain for cracks under transient loading, Acta Mechanica, 2016, 227(11), 3305-3314.

[24] M. Wuensche, Ch. Zhang, J. Sladek, V. Sladek, S. Hirose, M. Kuna, Transient dynamic analysis of interface cracks in layered anisotropic solids under impact loading, International Journal of Fracture, 2009, 157, 131-147.

[25] E. Lira-Vergara, C. Rubio-Gonzalez, Dynamic stress intensity factor of interfacial finite cracks in orthotropic materials, International Journal of Fracture, 2005, 135, 285-309.

[26] D.H. Li, W.H. Tai, Elastodynamic response of an interface crack in a layered composite under anti-plane shear impact load, Engineering Fracture Mechanics, 1991, 39, 687-93. 
[27] D.K. Shin, J.J. Lee, Fracture parameters of interfacial crack of bimaterial under impact loading, International Journal of Solids and Structures, 2001, $38,5303-5322$.

[28] S. Ueda, Y. Shindo, A. Astumi, Torsional impact response of a pennyshaped crack on a bimaterial interface, Engineering Fracture Mechanics, 1983, 18, 1059-1066.

[29] S. Karan, S. Basu, S.C. Mandal, Impact of a torsional load on a pennyshaped crack sandwiched between two elastic layers embedded in an elastic medium, Acta Mechanica, 2018, 229, 1759-1772.

[30] A.N. Guz, V.V. Zozulya, Elastodynamic unilateral contact problems with friction for bodies with cracks, International Applied Mechanics, 2002, 38, 895-932.

[31] M. Abramowitz, I.A. Stegun, Handbook of Mathematical Functions with Formulas, Graphs, and Mathematical Tables, Dover Publications, 1964.

[32] J. Sladek, V. Sladek, Dynamic stress intensity factors studied by boundary integro-differential equations, International Journal of Numerical Methods in Engineering, 1986, 32, 919-928.

[33] M.V. Menshykova, O.V. Menshykov, I.A. Guz, Modelling crack closure for an interface crack under harmonic loading, International Journal of Fracture, 2010, 165, 127-134.

[34] O.V. Menshykov, V.A. Menshykov, I.A. Guz, Elastodynamics of a crack on the bimaterial interface, Engineering Analysis with Boundary Elements, 2009, 33(3), 294-301. 
[35] A.N. Guz, I.A. Guz, O.V. Menshykov, V.A. Menshykov, Penny-shaped crack at the interface between elastic half-spaces under the action of a shear wave, International Applied Mechanics, 2009, 45(5), 534-539.

[36] O.V. Menshykov, M.V. Menshykova, W.L. Wendland, On use the Galerkin method to solve the elastodynamic problem for a linear crack under normal loading, International Applied Mechanics, 2005, 41(11), 1324-1329.

[37] S. Balas, J. Sladek, V. Sladek, Stress analysis by the boundary element methods, Amsterdam, Elsevier, 1989.

[38] M.H. Aliabadi, D.P. Rook, Numerical fracture mechanics, Computational Mechanics Publications and Kluwer Academic Publishers, 1991.

[39] O.V. Menshykov, M.V. Menshykova, I.A. Guz, Effects of crack closure and friction for linear crack under normal impact, Engineering Analysis with Boundary Elements, 2020, 115, 1-9. 